\title{
Crossed-Ratchet Effects for Magnetic Domain Wall Motion
}

\author{
A. Pérez-Junquera, ${ }^{1}$ V. I. Marconi, ${ }^{2}$ A. B. Kolton, ${ }^{2}$ L. M. Álvarez-Prado, ${ }^{1}$ Y. Souche, ${ }^{3}$ A. Alija, ${ }^{1}$ M. Vélez, ${ }^{1, *}$ J. V. Anguita, ${ }^{4}$ \\ J. M. Alameda, ${ }^{1}$ J. I. Martín, ${ }^{1}$ and J. M. R. Parrondo ${ }^{2}$ \\ ${ }^{1}$ Departamento de Física, Universidad de Oviedo-CINN, 33007 Oviedo, Spain \\ ${ }^{2}$ Departamento de Física Atómica, Molecular y Nuclear, and GISC, Universidad Complutense, 28040 Madrid, Spain \\ ${ }^{3}$ Institut Néel, Département NANO, CNRS and Universite Joseph Fourier, BP 166, 38042 Grenoble, France \\ ${ }^{4}$ Instituto de Microlectrónica de Madrid (CNM-CSIC), Tres Cantos, 28760 Madrid, Spain
}

(Received 31 August 2007; published 23 January 2008)

\begin{abstract}
We study both experimentally and theoretically the driven motion of domain walls in extended amorphous magnetic films patterned with a periodic array of asymmetric holes. We find two crossedratchet effects of opposite sign that change the preferred sense for domain wall propagation, depending on whether a flat or a kinked wall is moving. By solving numerically a simple $\phi^{4}$ model we show that the essential physical ingredients for this effect are quite generic and could be realized in other experimental systems involving elastic interfaces moving in multidimensional ratchet potentials.
\end{abstract}

The propagation of domain walls in thin ferromagnetic films is a problem of great current interest. It provides both the basis for a wide number of modern magnetic devices [1] and an excellent experimental system to study the basic physics of an elastic interface in the presence of either ordered or random pinning defects [2-6]. Such physics has been indeed recently considered in many other experimental systems involving interfaces, such as ferroelectric domain walls [7], contact lines of liquids menisci [8], or fractures [9]. Furthermore, it is relevant for systems involving periodic elastic manifolds, such as vortex lattices in superconductors [10], charge density waves [11] or Wigner crystals [12].

A case of particular interest appears when the pinning potential is asymmetric, favoring the propagation of the elastic interface in one direction. This gives rise to several ratchet effects [13], which are a potential tool to control motion at micro- and nanoscales in a variety of systems [14]. One of the first examples of ratchet potentials in magnetism is the use of "angelfish" patterns for controlling the sense of propagation of bubble domains in shift registers [15]. Much more recently, the asymmetric motion of domain walls (DWs) in triangular [16] or notched [17] nanowires has also been reported. In all previous cases, DW propagation is restricted to a narrow 1D path (either by narrow guide rails or by the nanowire geometry) and its transverse wandering can be neglected. Then the wall behaves essentially as a point particle in a 1D asymmetric potential. However, in a thin extended film, a DW is an elastic line that can distort all along its length in response to the $2 \mathrm{D}$ asymmetric pinning potential. The competition between elasticity and pinning is a purely collective behavior and can thus yield novel ratchet phenomena in 2D.

In this Letter, we study the propagation of DWs in extended magnetic films patterned with a periodic array of asymmetric holes. We observe experimentally, for the first time, two crossed-ratchet effects of opposite sign that change the preferred sense for DW motion depending on whether a flat or a kinked wall is moving. These crossed effects have an interesting consequence with potential applications: the system keeps memory of the sign of the last saturating state even in a zero magnetization configuration. Finally, by identifying the essential physics of the ratchet effect using a generic $\phi^{4}$ model, we show that this effect could be realized in other multidimensional ratchet systems involving the motion of elastic interfaces.

Amorphous $40 \mathrm{~nm}$ thick magnetic Co-Si films have been fabricated by sputtering with a well defined uniaxial anisotropy and a low coercivity [18]. In these films, easy axis (EA) magnetization reversal takes place by propagation of $180^{\circ}$ Néel walls that tend to lie parallel to the EA [18]. A $500 \times 500 \mu \mathrm{m}^{2}$ ordered array of asymmetric antidots has been patterned by a combination of $e$-beam lithography and an $\mathrm{Ar}^{+}$etching process [18]. Each hole is shaped as a small arrow pointing perpendicular to the EA [Fig. 1(a)]. Two senses of propagation for a DW lying along the EA ( $Y$ axis) may be defined: "forward" $(\mathcal{F})$, from left to right, i.e., towards the direction pointed by the arrows, and "backward" $(\mathcal{B})$, from right to left. The asymmetric anti-
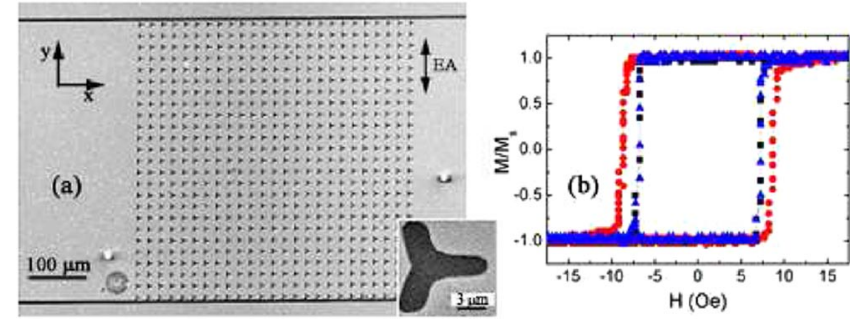

FIG. 1 (color online). (a) Scanning electron microscopy image of an array of asymmetric holes. The EA direction is indicated. The inset is a detail of a single arrow hole. (b) MOKE hysteresis loops measured at the array (circles) and at the left and right (triangles and squares) unpatterned regions. 
dots are arranged in a square array parallel to the EA, with a $20 \times 20 \mu \mathrm{m}^{2}$ unit cell, centered in a $500 \mu \mathrm{m}$ wide path and separated from the rest of the film by a $5 \mu \mathrm{m}$ wide trench. Magnetic properties have been characterized both by transverse magnetooptical Kerr effect (MOKE), using a setup with a laser focused in a $300 \mu \mathrm{m}$ spot in the desired sample area [18], and by MOKE microscopy [19]. The magnetic field $H$ is applied parallel to the film plane and along the EA.

Figure 1(b) shows hysteresis loops measured both at the array area (circles) and at the unpatterned regions at the left (triangles) and right (squares) sides of the array. The coercive field $\left(H_{C}\right)$ increases from 6.5 Oe in the continuous film to 8.6 Oe in the array, indicating that the arrow holes act as effective pinning centers, useful to control the DW motion. This difference in coercivity implies that there is a field range, approximately between 6.5 and $8 \mathrm{Oe}$, where the continuous regions have been reversed but not the patterned area, which will be bounded by two DWs at its left and right sides. This is indeed observed in the Kerr microscopy images shown in Figs. 2(a) and 2(b) taken at $H=8$ Oe after saturating the sample with a large negative field. A DW can be identified in each image as the line separating the dark-clear contrast regions (i.e., negative and positive magnetization). The walls are located either at the first [Fig. 2(a)] or at the last [Fig. 2(b)] column of defects, indicating that they cannot move further inside the array area due to the antidot pinning. Upon increasing the field to $H=8.4 \mathrm{Oe}$, the left wall penetrates the array [see Fig. 2(c), in which the left wall is pinned between the 4th and 5th antidot columns]. Then, at $H=8.8$ Oe [Fig. 2(d)] the left wall has propagated in the $\mathcal{F}$ direction, up to the 17 th defect column, whereas the right wall has not been yet able to move. Finally, for larger fields, both walls coalesce completing the magnetization reversal at the array area [Fig. 2(e)]. A similar image sequence for the descending field branch in the hysteresis loop shows again a wall entering from the left and moving in the $\mathcal{F}$ direction, only with an overall magnetization sign change. These reversal sequences clearly show that the depinning field

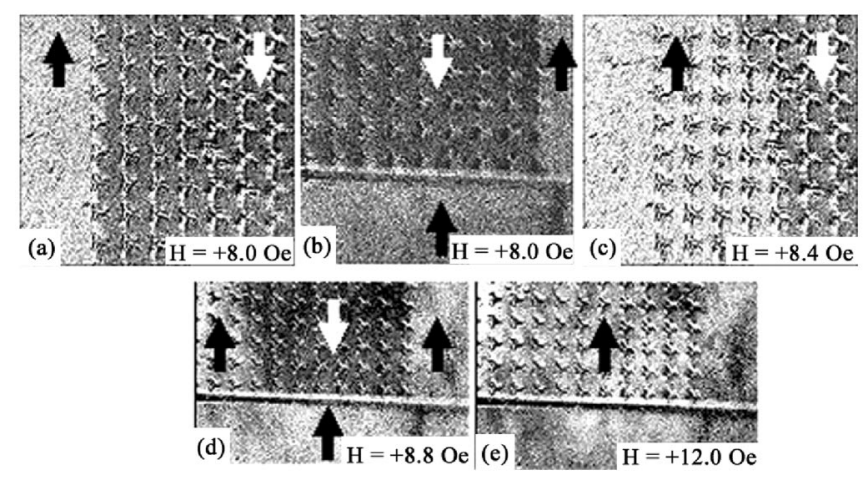

FIG. 2. (a)-(e) Sequence of MOKE microscopy images taken in the ascending field branch of the hysteresis loop after saturation in a negative field. for $\mathcal{F}$ wall propagation $H_{\mathcal{F}} \approx 8.4$ Oe is lower than the field for $\mathcal{B}$ wall depinning $H_{\mathcal{B}} \geq 8.8$ Oe (only a lower bound can be obtained in this case) indicating that the arrow-shaped holes act as asymmetric pinning centers for the DWs. The easy direction of motion $\mathcal{F}$ is that in which the length of the pinned wall between two antidots increases smoothly, in agreement with the reported behavior in nanowires of triangular cross section [16,17]. Preliminary micromagnetic simulations with the OOMMF code [20] also confirm this behavior [21].

To study the motion of a single DW inside the array, the following experiment has been performed [see $H(t)$ sequence in Fig. 3(a)]: first, to introduce a DW in the array, a complete major loop is performed between $t=0$ and $t=$ $0.25 \mathrm{~s}$; then, the sample is saturated in a large negative field; next, $H$ is increased up to the positive coercivity, so that the DW enters into the array, and then $H$ is decreased. Now, at $t=t_{0}$, a triangular field ramp of increasing amplitude $H_{\max }$ is applied to the sample so that the DW is pushed $\mathcal{F}$ and $\mathcal{B}$. The magnetization response $M(t)$ is shown at the bottom of Fig. 3(a), both in the patterned and unpatterned regions. Surprisingly, during several cycles (between $t_{0}$ and $t_{1}$ ), there is a net decrease in $M$ at the array [see inset of Fig. 3(a)], indicating $\mathcal{B}$ DW motion. The sign of the $\partial M / \partial t$ slope at $t=t_{0}$ depends only on the sign of the saturation magnetization $M_{S}$ before introducing the wall in the array (inset of Fig. 3), and not on the sign of $\partial H / \partial t$, as would be in a standard accommodation effect [22]. Figure 3(b) shows several stable minor loops, measured with a similar $H(t)$ as in Fig. 3(a), but with a constant amplitude $H_{\max }<8$ Oe in the triangular ramp after $t_{0}$ and

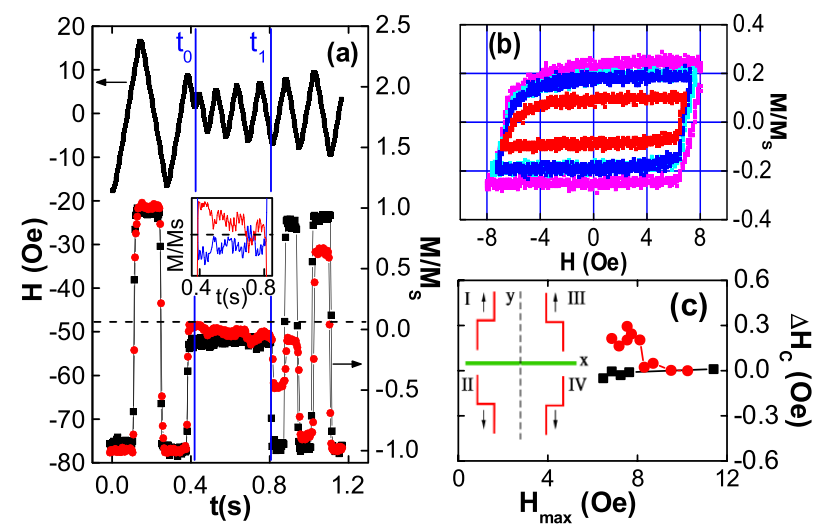

FIG. 3 (color online). (a) $H(t)$ used to introduce a wall in the array and measure its propagation within it (top); $M(t)$ response in the array (red circles) and in the continuous film (black squares). The inset is an enlargement of the decrease (increase) in $M(t)$ from $t_{0}$ to $t_{1}$ in the array after negative (positive) saturation [top or red (bottom or blue) line]. (b) Minor loops measured after introducing a wall in the array at the positive $H_{C}$. (c) Coercive field asymmetry vs. minor loop amplitude in the array (red circles) and in the continuous film (black squares). The inset shows the results of $X$ axis reflection and $Y$ axis reflection (broken symmetry in the array) on a kink moving upward. 
centered along the magnetization axis. These minor loops exhibit a clear asymmetry, quantified by the difference between the positive and negative coercivities $\Delta H_{C}=$ $H_{C}^{\text {asc }}-H_{C}^{\text {desc }}$ of about 0.2 Oe [Fig. 3(c)]. Different from exchange bias, the sign of $\Delta H_{C}$ depends on the sign of $M_{S}$ before introducing the DW, so that coercivity is always lower when the DW is pushed in the $\mathcal{B}$ direction.

From these data, two main results are worth remarking: first, the system keeps memory of the last saturating state that can be read in the sign of $\Delta H_{C}$ or of $\partial M / \partial t$ at $t=t_{0}$. Second, there is a clear change between the behavior observed in Fig. 2, in which the DW penetrates into the array more easily in the $\mathcal{F}$ direction, and the minor loop experiment of Fig. 3, in which DW motion within the array is easier in the $\mathcal{B}$ direction.

To understand these opposite effects, a crucial observation is the change in the wall configuration as it enters the array: in the continuous area, the wall is essentially flat [Figs. 2(a) and 2(b)] but it develops kinks when it is pinned into the array [Fig. 2(c)]. This suggests an extra mechanism for DW motion in the minor loop experiment, through upward (downward) $[\mathcal{U}(\mathcal{D})]$ kink propagation, that is possible in our geometry but not in the more restricted nanowire case where the DW cannot develop kinks. Indeed, by taking into account the reflection symmetries of the array as depicted in the inset of Fig. 3(c), it is easy to see that a kink propagating $\mathcal{U}$ (I) would be equivalent to an antikink propagating $\mathcal{D}$ (II) but not necessarily equivalent to a kink propagating $\mathcal{D}$ (IV) or to an antikink propagating $\mathcal{U}$ (III) (see also the insets in Fig. 4). Therefore, the array could in principle induce an asymmetric pinning for kink motion, i.e., perpendicular to the previously described ratchet affecting the $\mathcal{F} / \mathcal{B}$ propagation of the flat wall

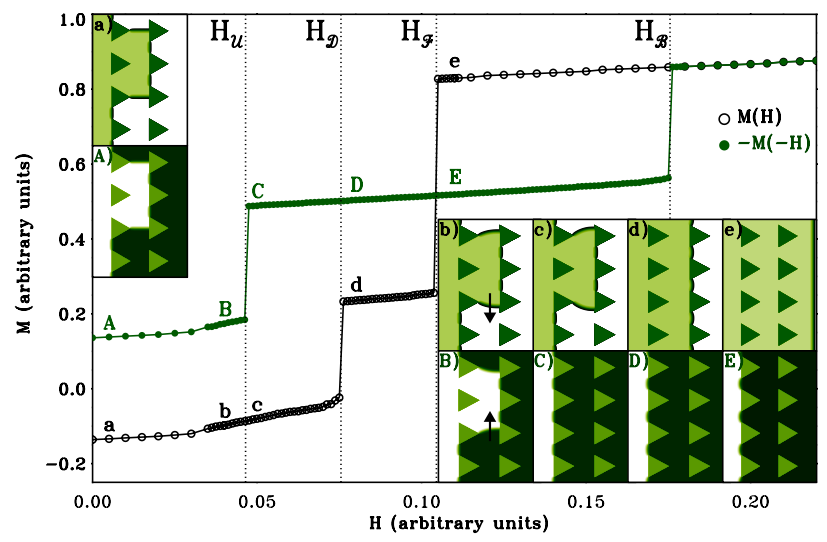

FIG. 4 (color online). Numerical results for the magnetic response of a kinked domain wall vs applied magnetic field. The initial state at $H=0$ is a wall with a kink (antikink) pair [insets (a) and (A)], which evolves asymmetrically with respect to $H$ and $-H$. The insets are snapshots of the local magnetization $\phi$, for different pairs of fields of equal magnitude, marked in the magnetization curves as $a, \ldots, e$, in $M(H)$, and as $A, \ldots E$ in $-M(-H)$. Critical fields show that $\mathcal{F}$ motion is easier for a flat wall but $\mathcal{B}$ motion is easier for the kinked wall.
[23]. Most interestingly, the experimental results of Figs. 2 and 3 suggest that these crossed-ratchet effects must be of opposite sign.

To check the above scenario we consider the competition between drive, elasticity, and asymmetric pinning on a single driven DW. For this purpose we simulated the paradigmatic $\phi^{4}$ model for a scalar order parameter $\phi(x, y ; t)$, in which a DW provides a smooth transition between energetically equivalent minima of a simple free energy [24]. We will show that this approach, although simplistic as it avoids many of the complications of the full micromagnetic model, qualitatively reproduces the experimental results. Consequently, the aforementioned competition between drive and elasticity of the DW, which is an essentially $2 \mathrm{D}$ feature absent in previous $1 \mathrm{D}$ works and that is fully captured by the $\phi^{4}$ model, is the main physical ingredient for the effect. Most importantly, reproducing the effect in the $\phi^{4}$ model allows us to demonstrate the general nature of the observed ratchet phenomena. In our model $\phi(x, y ; t)$ can be thought as a projection of the coarsegrained magnetization vector along the easy direction. We consider the evolution of $\phi$ in the domain $\Omega-\triangle$, which includes all the space $\Omega$, except the region $\triangle$ occupied by antidots. In order to model the absence of magnetic material in $\triangle$, we set Neumann boundary conditions $\left.\partial_{\mathbf{n}} \phi\right|_{\partial \triangle}=0$ at the antidot borders $\partial \triangle$. Finally, considering a purely dissipative dynamics, the equation of motion for $\phi$ reads [24]

$$
\eta \partial_{t} \phi=c \nabla^{2} \phi+\epsilon_{0}\left(\phi-\phi^{3}\right)+H,
$$

where $c$ is the elastic stiffness of the order parameter, $\epsilon_{0}$ is proportional to the local barrier separating the two equivalent minima of the free energy density, $H$ represents the magnetic field, and the friction coefficient $\eta$ sets the microscopic time scale. The relevant parameters $c$ and $\epsilon_{0}$ will determine both the width $\xi \propto \sqrt{c / \epsilon_{0}}$ and the line tension $\sigma \propto \sqrt{c \epsilon_{0}}$ of the DW [24].

For the simulation we chose $\epsilon_{0}=\eta=1$ and $c$ such that $\xi$ is $10 \%$ of the characteristic size of the antidots, which approximately corresponds to the realistic situation [18], although we obtain qualitatively the same behavior for a finite range of parameters. We solve numerically Eq. (1) in a $L_{x} \times L_{y}$ box with periodic boundary conditions in the $Y$ direction, and model the asymmetrical antidots as a rectangular array of triangular holes pointing to the positive $X$ direction (see insets in Fig. 4). To ensure the presence of a DW along the sample, we set $\phi(x=0, y ; t)=1$ and $\phi\left(x=L_{x}, y ; t\right)=-1$ as boundary conditions in the $X$ direction, and then probe the response of the DW to different, positive and negative $H$. Since we are interested in the response to constant or low-frequency fields, we will only analyze the stationary magnetization $M(H)$, starting with the particular initial condition of a single flat DW with a kink (antikink) pair (see insets A and a in Fig. 4).

In Fig. 4 we show the magnetization $M$ vs $H$ starting at $H=0$ with the kink (antikink) pair [21]. As indicated by 
the vertical dotted lines we can clearly distinguish four critical fields: $H_{\mathcal{U}}$ corresponds to the $\mathcal{U}(\mathcal{D})$ depinning of a kink (antikink), cases I and II in Fig. 3(c), which amounts to a net motion of our initial DW to the left $(\mathcal{B}) ; H_{\mathcal{D}}$ is the depinning field for the $\mathcal{D}(\mathcal{U})$ motion of a kink (antikink), cases IV and III in Fig. 3(c); $H_{\mathcal{F}}$ and $H_{\mathcal{B}}$ corresponding to the $\mathcal{F}$ and $\mathcal{B}$ depinning fields of flat walls, respectively. We find that $H_{\mathcal{U}}<H_{\mathcal{D}}<H_{\mathcal{F}}<H_{\mathcal{B}}$. As expected, transport at low fields $|H|<H_{\mathcal{F}}$ is dominated by the presence of mobile kinks. More interestingly, we have $H_{\mathcal{U}}<H_{\mathcal{D}}$. This implies that a net directed transport of the wall in the $\mathcal{B}$ direction can be obtained under a low-frequency ac field of amplitude $H_{U}<|H|<H_{\mathcal{D}}$, in qualitative agreement with the behavior of the magnetization in the minor loop experiment (Fig. 3). Finally, by increasing the magnetic field amplitude $|H|$ we have an inversion of the rectification for flat walls, since $H_{\mathcal{B}}>H_{\mathcal{F}}$, i.e., the wall as a whole moves more easily in the $\mathcal{F}$ direction, as in the experiments (cf. Fig. 2). The simulations also show which are the key ingredients for the inversion in the rectification between these two crossed-ratchet effects: whereas the $\mathcal{B}$ motion of a flat wall (Fig. 4, inset $\mathrm{E}$ ) involves a sudden (i.e., long-range correlated) depinning from the stable position at the base of the triangles, making it the hard direction of motion, the $\mathcal{B}$ motion of a kinked wall involves the $\mathcal{U}$ motion of a kink (Fig. 4, inset B), which gradually peels off the wall from the triangle bases thus making this the easy direction of motion for a kinked wall. It is worth noting that this behavior is due to the generic interplay between elasticity, pinning, and drive: while the first two tend to minimize the line energy of the DW by, respectively, straightening all segments and by optimally using the holes bridging them, the applied field tends to increase the area behind the DW with $\phi H>0$. This leads to the asymmetric depinning configurations and forces, responsible for the observed crossed-ratchet effects.

In summary, our experimental and theoretical study of the DW propagation across an array of asymmetric holes has revealed the existence of two crossed-ratchet effects: the first one favors $\mathcal{F}$ motion of a flat wall while the second acts on the $\mathcal{U}$ or $\mathcal{D}$ kink propagation favoring net $\mathcal{B}$ wall motion at low fields. As a result of the interplay between both ratchets, the system keeps memory of the sign of the last saturating state even in a zero magnetization configuration, thus opening an interesting possibility for future applications in memory devices. This novel effect relies completely in the extended nature of the DW in 2D, which allows excitations transverse to the direction of propagation, and not on the particular asymmetry of the pinning potential. Moreover, the main physical ingredient for the crossed ratchets can be identified as the interplay between elasticity, asymmetric pinning, and drive, so that they could be realized in other experimental systems involving the motion of elastic interfaces or domain walls in multidimensional ratchet potentials.
Work supported by Spanish CICYT under Grants No. NAN2004-09087, No. MOSAICO, and No. FIS200507392. We acknowledge helpful discussions with Professor J.L. Vicent.

*mvelez@uniovi.es

[1] C. D. Mee and E. D. Daniel, Magnetic Storage Handbook (McGraw-Hill, New York, 1996); G. Prinz, J. Magn. Magn. Mater. 200, 57 (1999).

[2] M. Kardar, arXiv:cond-mat/9704172; T. Giamarchi, A. B. Kolton, and A. Rosso, in Jamming, Yielding, and Irreversible Deformation in Condensed Matter, edited by M. C. Miguel and J.M. Rubi, Lecture Notes in Physics Vol. 688 (Springer, New York, 2006), p. 91.

[3] S. Lemerle et al., Phys. Rev. Lett. 80, 849 (1998).

[4] J. P. Attane et al., Phys. Rev. Lett. 93, 257203 (2004).

[5] Y. L. Iunin et al., Phys. Rev. Lett. 98, 117204 (2007).

[6] E. Martinez et al. Phys. Rev. Lett. 98, 267202 (2007).

[7] P. Paruch, T. Giamarchi, and J. M. Triscone, Phys. Rev. Lett. 94, 197601 (2005).

[8] S. Moulinet et al., Phys. Rev. E 69, 035103(R) (2004).

[9] L. Ponson, D. Bonamy, and E. Bouchaud, Phys. Rev. Lett. 96, 035506 (2006).

[10] G. Blatter et al., Rev. Mod. Phys. 66, 1125 (1994).

[11] T. Nattermann and S. Brazovskii, Adv. Phys. 53, 177 (2004).

[12] T. Giamarchi, Electronic Glasses (IOS Press, Bologna, 2004); arXiv:cond-mat/0403531.

[13] P. Reimann, Phys. Rep. 361, 57 (2002); R. D. Astumian and P. Hänggi, Phys. Today 55, No. 11, 33 (2002).

[14] J. E. Villegas et al., Science 302, 1188 (2003); H. Linke et al., Science 286, 2314 (1999); D. E. Shalóm and H. Pastoriza, Phys. Rev. Lett. 94, 177001 (2005); C. C. de Souza Silva et al., Nature (London) 440, 651 (2006).

[15] N. Hayashi et al., IEEE Trans. Magn. 8, 370 (1972).

[16] D. A. Allwood, G. Xiong, and R. P. Cowburn, Appl. Phys. Lett. 85, 2848 (2004).

[17] A. Himeno et al., J. Appl. Phys. 97, 066101 (2005); M. Hayashi et al., Phys. Rev. Lett. 97, 207205 (2006); S. Savel'ev, A. Rakhmanov, and F. Nori, New J. Phys. 7, 82 (2005).

[18] M. Vélez et al., Eur. Phys. J. B 41, 517 (2004); A. PérezJunquera et al., J. Appl. Phys. 99, 033902 (2006).

[19] C. Coquaz et al., J. Magn. Magn. Mater. 124, 206 (1993).

[20] Object Oriented Micromagnetic Framework is available at http://math.nist.gov/oommf.

[21] See EPAPS Document No. E-PRLTAO-100-037802 for movies of simulations of DW motion at fixed magnetic fields. For more information on EPAPS, see http://www. aip.org/pubservs/epaps.html.

[22] P. J. Thompson and R. Street, J. Phys. D 30, 1273 (1997).

[23] Note that any net $\mathcal{U}$ or $\mathcal{D}$ pressure on the kinked DW due to small field misalignment would be symmetric upon $X$ axis reflection and does not introduce any extra asymmetry in the problem.

[24] See, for instance, P. M. Chaikin and T. C. Lubensky, Principles of Condensed Matter Physics (Cambridge University Press, Cambridge, 1995). 